\title{
Microscale Effects From Global Hot Plasma Imagery
}

\author{
T. E. Moore and M.-C. Fok \\ Space Sciences Laboratory, NASA Marshall Space Flight Center, Huntsville, Alabama
}

J. D. Perez and J. P. Keady

Department of Physics, Auburn University, Auburn, Alabama

\begin{abstract}
We have used a three-dimensional model of recovery phase storm hot plasmas to explore the signatures of pitch angle distributions (PADs) in global fast atom imagery of the magnetosphere. The model computes mass, energy, and position-dependent PADs based on drift effects, charge exchange losses, and Coulomb drag. The hot plasma PAD strongly influences both the storm current system carried by the hot plasma and its time evolution. In turn, the PAD is strongly influenced by plasma waves through pitch angle diffusion, a microscale effect. We report the first simulated neutral atom images that account for anisotropic PADs within the hot plasma. They exhibit spatial distribution features that correspond directly to the PADs along the lines of sight. We investigate the use of image brightness distributions along tangent-shell field lines to infer equatorial PADs. In tangent-shell regions with minimal spatial gradients, reasonably accurate PADs are inferred from simulated images. They demonstrate the importance of modeling PADs for image inversion and show that comparisons of models with real storm plasma images will reveal the global effects of these microscale processes.
\end{abstract}

\section{INTRODUCTION}

Microscale processes in a plasma are those having scales larger than the Debye length, but smaller than any other relevant scales in the plasma system or subsystems. The term also connotes "collective effects" as contrasted with collisional effects that occur on atomic collision scales. These microscale processes are thought to reduce the mean free path associated with the collisional effects, augmenting those weak effects in nearly collisionless space plasma systems. In such a case, microscale processes have the shortest time scales in the system.

There has been a long and honorable debate between those who advocate the importance of such microscale collective effects and those who are skeptical of their significance. The skeptics have argued convincingly that

Cross-Scale Coupling in Space Plasmas

Geophysical Monograph 93

Copyright 1995 by the American Geophysical Union simple observation of microscale effects, which appear ubiquitous in space plasmas, is necessary but not sufficient to demonstrate that they have important consequences. They argue that all collisional effects must be fully evaluated and shown to be inadequate before a solid case can be made for significant microscale effects. The result has been a standoff in which the advocates have been led to state their results in terms of possibilities instead of certainties, e.g. "plasma waves can cause enhanced particle loss through diffusion into the loss cone." If space physics is to become a predictive science capable of supporting an operational space weather service, we must overcome this problem and reach the point where we know that microscale effects will produce specific consequences.

One approach that is being taken is to more rigorously and completely evaluate the actual effects of collisional processes in space plasmas. For example, Fok et al. [1995] have developed a fairly detailed model of the effects of charge exchange and Coulomb drag on the storm time, recovery phase hot plasmas of the inner magnetosphere. This model tracks the losses from the storm's hot plasma system during recovery and therefore leads to specific 
comparisons between the observed and modeled recovery phase plasma evolution. Preliminary indications from this comparison are that the modeled hot plasma decays more gradually in the early phase than the observed hot plasma does, as measured by the $D_{\text {st }}$ index of the storm ring current. One hypothesis concerning this discrepancy is that plasma wave-driven pitch angle diffusion causes higher loss rates in the real world than in the model, which does not account for such microscale effects. In this paper, we identify a plan for evaluating this hypothesis in some detail, using observations that would be provided by a fast atom-imaging instrument, as proposed for the Magnetosphere Imager Mission being studied by Marshall Space Flight Center for the NASA Space Physics Division.

\section{THREE-DIMENSIONAL HOT PLASMA MODEL AND HOT PLASMA IMAGING}

The storm-time plasma model used herein is that of Fok et al. [1995], to which the reader is referred for additional details. In brief, the model solves a Boltzmann initial/boundary value problem with specified electric and magnetic fields, including the effects of charge exchange losses and Coulomb drag on energetic particles interacting with the hydrogen geocorona and the plasmasphere, respectively. Rather than tracking particle trajectories in detail, a bounce-averaged approach is taken. At present, a dipole magnetic field has been used for the region between 2.0 and $6.5 R_{E}$. The circulation electric field is prescribed by the Volland-Stern model, and the plasmasphere is from the model of Rasmussen et al. [1993], with variations prescribed by the time series of $K_{p}$ values for any specific storm interval. The initial and outer boundary conditions are derived from observations of storm main phase hot plasmas, i.e., those of Hamilton et al. [1988]. The main contribution of this storm plasma model lies in its attention to the pitch angle-dependent effects of charge exchange and Coulomb collisions. It computes the equatorial pitch angle distributions (PADs) as a function of ion species and location in the equatorial plane, leading to an effective three-dimensional description of the hot plasma distribution in space.

As compared with other recent simulations [e.g., Roelof et al., 1985; Williams et al. 1992; Moore et al., 1992] of fast neutral atom emission from the storm plasma region, the present work adds a realistic description of the spatial and temporal evolution of the hot plasma in three dimensions. The charge exchange reaction is essentially free of angular scattering, so the angular distribution of fast atoms emitted from each volume of space is essentially identical to the angular distribution of the parent ion population. At energies of tens of $\mathrm{eV}$ and higher, emitted fast atoms travel essentially in straight lines and form a basis for imaging the emissivity distribution from arbitrary vantage points. It is apparent that isotropic hot plasma produces a different flux brightness distribution than anisotropic hot plasma. The brightness distribution of an image is thus influenced as much by the (pitch) angular distribution of the hot plasma as it is by its spatial distribution.

It has been supposed that knowledge of the geocoronal hydrogen distribution allows it to be deconvolved from the fast atom images, yielding the spatial distribution of the hot plasma ions. However, as with most inverse problems, the solution is never unique. Selection of a credible solution from among the nonunique possibilities depends upon the availability and accuracy of other knowledge of the form of the hot plasma distribution. For example, the inversion is vastly improved when imagery is available from multiple complementary vantage points, permitting tomographic inversion. All such inversions proceed from forward modeling of the hot plasma distribution that must, at a minimum, include all parameters that significantly influence the images. The arguments above mandate that these parameters must in any case include a reasonable description of the possible hot plasma pitch angle distributions.

Acceleration and transport processes produce freshly injected storm plasmas ranging from nearly isotropic to "cigar-shaped," having magnetic field-aligned angular distributions. Charge exchange and Coulomb losses owing to interactions with the terrestrial neutral atmosphere remove ions most rapidly at field-aligned pitch angles, causing the equatorial distributions to become deficient in such particles, or "pancake" shaped. The ensuing loss of storm particles is very strongly influenced by the rate of pitch angle diffusion, which transports particles from long-lasting equatorial mirroring pitch angles to smaller angles, resulting in much more rapid loss. In turn, the rate of pitch angle diffusion is controlled by the amplitude of plasma waves that are driven by the pitch angle anisotropy and other microphysical features of the plasma. The actual form of the PAD is more isotropic than it would be in the absence of such waves, to a degree that is indicative of the intensity spectrum of the waves.

Clearly, proper accounting for the possible angular distributions within storm plasmas adds to the complexity of the image inversion process. On the other hand, no inversion that ignores angular distributions can conclusively identify spatial features of the hot plasma. Image inversions that properly account for PADs hold the promise to provide evidence of microscale, as well as macroscale, processes operative during storm recovery. In this paper, we assess the degree to which fast atom imagery can support the evaluation of microscale effects on storm recovery using an appropriate model. The three-dimensional driftloss model provides the basis for simulating the flux of fast neutral atoms emitted as a result of the interaction of the storm-time plasmas with the hydrogen geocorona and plasmasphere, in the absence of pitch angle diffusion. However, the goal will be to identify means for determining, from real images, the true PADs of the hot plasma ions. To the degree that the inferred PADs are more 
isotropic than those predicted by this model, the results will indicate the presence of significant plasma wave intensities and resultant pitch angle diffusion.

To provide important global context, we begin with the simulation of polar views of fast atom emission from the storm recovery phase hot plasmas. A simple means for qualitative recovery of the integral ion flux is demonstrated using these images. However, storm plasma PAD features are expected to appear most clearly in the distribution of fast atom emissivity along selected field lines. Therefore, in subsequent sections we simulate equatorial views of the hot plasma in which equatorial and footpoint regions can be clearly distinguished. The same simple method for qualitative ion flux recovery is demonstrated on these images. Finally, a simple but quantitative method for inferring equatorial PADs is demonstrated. The results are discussed in terms of more advanced inversion techniques.

\section{POLAR VIEWS}

It is useful to gain a global perspective of the hot plasma distribution before focusing on the distribution along a particular field line. To gain such a global view of the hot storm plasmas, a view from high over the magnetic pole of the Earth is advantageous. Therefore we begin with development of polar views of the hot plasma.

\section{Integral Hot Plasma Ion Flux}

A useful step toward the production of simulated fast neutral atom images is the line-of-sight integration of the ion flux within a specified model distribution of magnetospheric hot plasma, without convolution with the geocoronal hydrogen distribution. Knowledge of the results absent the convolution are useful in the interpretation of fast atom images that incorporate the convolution. Plate 1 (left panel) shows the result of a line-of-sight integration of the ion flux from a polar vantage point, for protons of energy 1.7 $\mathrm{keV}$, at a time 6 hours into the recovery phase of the major storm that occurred in February, 1986. In this integration, the ion flux within each step along the line-of-sight is taken from the relevant modeled local PAD according to the local pitch angle of the line-of-sight. Consequently, image brightness is controlled not only by the spatial distribution of ion flux, but also by the local PAD according to the predominant orientation of the line-of-sight relative to the local magnetic field.

Notable features of the $1.7 \mathrm{keV}$ proton plasma that are evident from this image include the strong minimum of flux on the dayside, and the well-defined convecting cloud in the 0900LT sector (upper left of the left panel). We omit a similar image for the proton plasma at a higher energy of $100 \mathrm{keV}$, because the most notable feature would he the highly uniform distribution of flux in local time. This is a direct result of the very fast gradient and curvature drift of such energetic ions, and their relatively negligible convection drift speed.

\section{Fast Neutral Atom Flux}

When the cold atomic hydrogen geocoronal distribution is appropriately convolved into the polar images shown above, the differences (apart from absolute magnitudes), as shown in Plate 2, lie mainly in the radial distribution of emission, which responds to the strong peaking of geocoronal density close to the Earth. Because the ion fluxes are confined to magnetic field lines extending deep into the geocorona at high latitudes, the brightest emissions originate from the "footpoints" of the field lines, intersecting the Earth near the polar cap boundary. This produces the bright emission which appears inside the disk of the Earth indicated by the circle in these images.

We evaluate the fast neutral atom flux at 1-degree resolution for a hemispherical field of view, as shown in the left panel of Plate 2. In the right panel of Plate 2, we show the result of passing this flux through a simple instrument filter, specified by a pixel solid angle $(4 \times 4$ degree in this case), an effective area $\left(1 \mathrm{~cm}^{2}\right)$ ), and an accumulation time (600 sec., assuming the image is obtained by a single row of pixels on a spinning spacecraft, and thus applying a pixel accumulation duty cycle of 4 degree of each 360 . degree spin). The image is then expressed in counts for the accumulation, where integer counts have been selected at random from a range extending above and below the simulated value by one Poisson standard deviation, an approximate method for introducing counting noise.

\section{Recovery Of Hot Plasma Ion Flux}

Inspection of Plate 2 suggests that morphological features of the ion flux might be approximately recovered from the fast atom flux through a simple radial image rescaling to remove the radial dependence of the geocoronal hydrogen column density. Such a rescaling would implicitly assume that the geocoronal hydrogen and ion flux distributions along the line-of-sight are only weakly dependent on image pixel position, i.e., that they are factorable into separate line-of-sight integrations of the ion flux and the cold geocoronal density, forming separate ion flux and geocoronal images. To the extent that the ion flux is mainly confined to or largest at the equator, this assumption may be justifiable. With this simplifying assumption, the fast atom flux image $F_{N A}$ can be approximately related to the integral ion flux image, $F_{i}$ as follows:

$$
F_{N A}=\sigma \int_{L O S} n_{H} f_{i} d l \approx \sigma \frac{1}{L} \int_{L O S} n_{H} d l \cdot \int_{L O S} f_{i} d l=\sigma\left\langle n_{H}\right\rangle \cdot F_{i}
$$

This equation applies separately to each image pixel, $\sigma$ is the relevant cross section, $L$ is the length of the line-of- 


\section{Integral lon Flux [L $\left.\Omega T_{T E}\right]^{-1}$}

\section{Recovered Ion Flux}
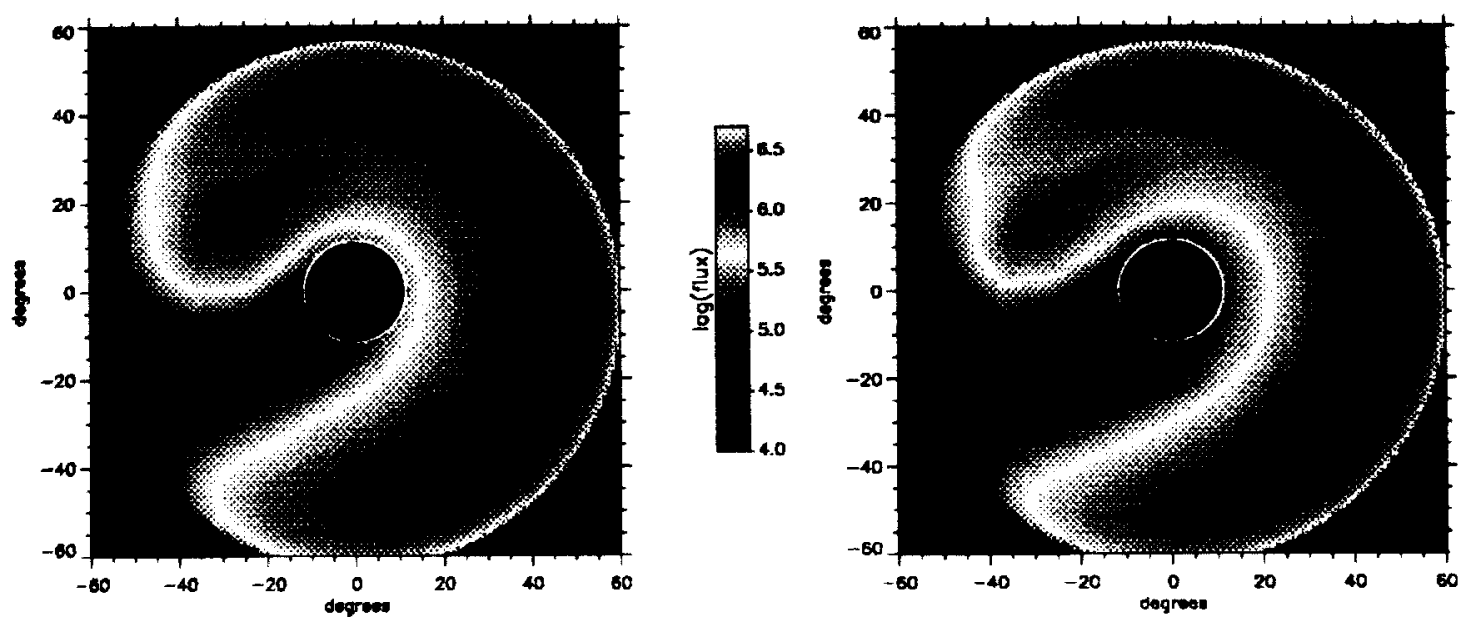

Plate 1. Comparison of model flux with flux as recovered from a simulated fast atom image. Left panel: the line-ofsight integral flux of $1.7 \mathrm{keV}$ protons, as a two-dimensional hemispherical function of look angle, from a vantage point $5 R_{E}$ over the north pole, with the Sun toward the left. The three-dimensional ion flux is derived from the model of Fok et al. [1995], for an instant of time 6 hours into the recovery phase of a major magnetic storm (Feb. $1986 \mathrm{storm}$ ). Right panel: an estimate of the integral flux of $1.7 \mathrm{keV}$ protons equivalent to the left panel, but derived through a simple recovery method from a simulated fast atom image of the same hot plasma population.

\section{Neutral Atom Flux $\left(\mathrm{cm}^{2} \mathrm{sr} \mathrm{s} \mathrm{keV}\right)^{-1}$}

\section{Image Counts}
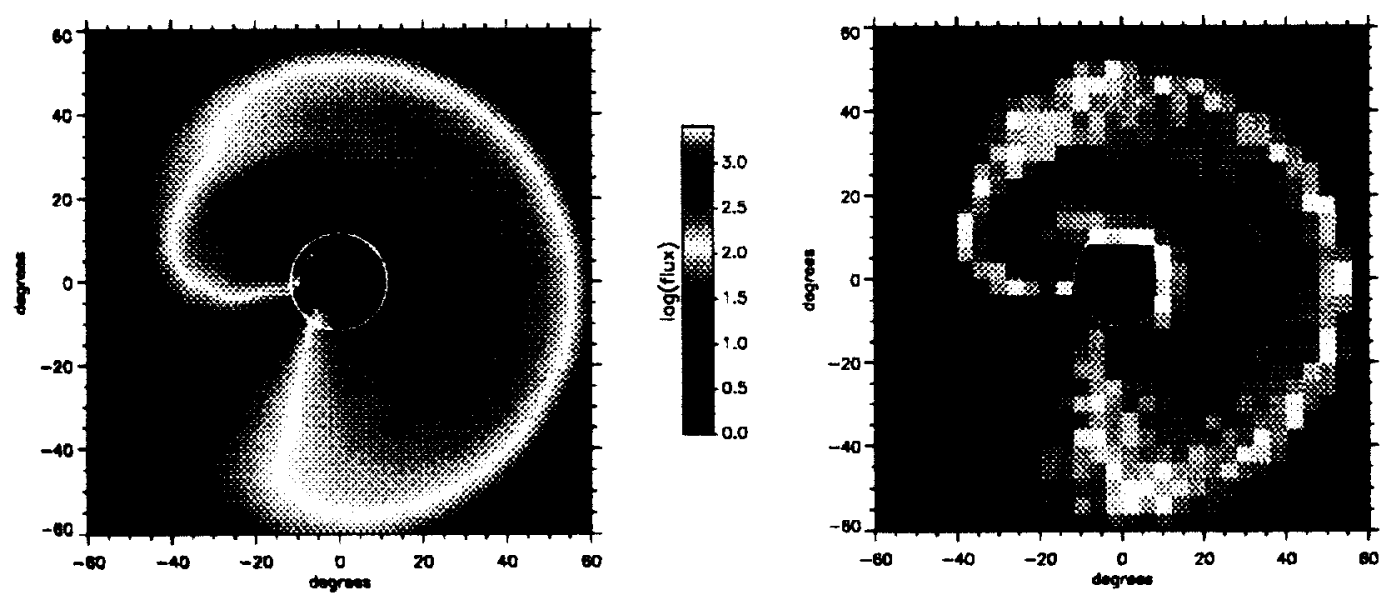

ongie resolution: $4^{\circ} \times 4^{\circ}$ Exposure: 600 100 Aeff: $1.00 \mathrm{~cm}^{2}$ oE: $\quad 3.00 \mathrm{keV}$

Plate 2. Comparison of the flux image of fast atom emission from the storm-time plasma, with the response to that flux of a hypothetical imaging instrument. Left panel: the fast atom image computed by the convolution of the ion flux distribution illustrated in Plate 1, left panel, with the geocoronal hydrogen density and the cross section for charge exchange between $1.7 \mathrm{keV}$ protons and cold hydrogen, leading to a fast hydrogen atom and a cold proton. Right panel: the response of an imaging instrument to the flux distribution of the left panel, assuming an aperture of $1 \mathrm{~cm}^{2}, 4 \times 4$ degree pixels, and an energy bandwidth of $3 \mathrm{keV}$. 
sight integration path, $n_{H}$ is the geocoronal density, $f_{i}$ is the ion flux in the viewing direction, and $F_{i}$ is the ion integral flux. The geocoronal image $\left\langle n_{H}\right\rangle$ is implicitly defined by the equation. The integral ion flux can then be approximately recovered from the neutral atom image $\left(F_{N A}\right)$, using the geocoronal hydrogen integral image, as follows:

$$
F_{i} \approx F_{N A} \frac{1}{\sigma\left\langle n_{H}\right\rangle}
$$

Plate 1 (right panel) shows the result for a polar view image of the magnetosphere at $1.7 \mathrm{keV}$. Recall that the left panel is the integral ion flux at 1 degree resolution. In the right panel is the quotient of the fast atom flux image at 1 degree resolution and the integral hydrogen density image, at the same resolution. It can be seen that the recovered ion flux is very similar to the model ion flux. Certainly, this technique provides a qualitatively credible reconstruction of the integral ion flux in this case.

This exercise is intended to demonstrate the plausibility of recovering meaningful ion flux distributions from fast atom imagery. It is clear that a more rigorous inversion will be required to quantitatively deconvolve the image, but this should not detract from the basic value of the method. Routine recovery of the images will yicld information that will be useful in a browse mode where images must be selected for more rigorous inversion and analysis, or as a basis for initial guesses in an iterative inversion process.

\section{EQUATORIAL VIEWS}

\section{Integral Hot Plasma Ion Flux}

Complementary to the polar imagery of this storm plasma model, Plate 3 (left panel) shows the integral ion flux of protons with $1.7 \mathrm{keV}$ energy at 6 hours recovery phase, but from a location at the equator near dusk local time and at a distance of $5 R_{E}$. Notable features of the image include highly structured PADs that lead to strong spatial structuring of the ion flux, with contrasting behavior in the two halves of the plate. The vantage point and storm phase have been chosen so that the left (dayside) half of the left pancl image reflects a region with "pancaked" PADs leading to equatorial peaking of the brightness, whereas the right (nightside) half of the image reflects a region with somewhat field-aligned PADs, leading to brightness maxima at the footpoints of the ficld lines in some locations. Since these are hemispherical allsky images, distances are difficult to judge. The projections of $L=2,4$, and 6 field lines are shown superposed on the image as a spatial reference system.

For comparison, Plate 4 (left panel) shows the integral ion flux from the same vantage point, but for protons of
$100 \mathrm{keV}$ energy, some 36 hours into the recovery phase. In this case, the ion flux is characterized by "pancaked" PADs on both day and night sides of the image with only modest spatial structuring of the ion flux or PAD. These characteristics reflect the late stage of the recovery phase, and the uniformity in local time that is expected for energetic ion populations with large drift speeds.

\section{Fast Neutral Atom Flux}

As in the case of polar views, the ion flux features are still generally discernible in the equatorial view of fast atom flux shown in the left side of Plates $5(1.7 \mathrm{keV})$ and 6 (100 keV). However, these features remain severely distorted by the cold hydrogen density distribution, which enhances the emissivity in regions close to the Earth. This is true of both the $1.7 \mathrm{keV}$ neutral atom images and the 100 $\mathrm{keV}$ images. The response of a hypothetical imaging instrument to these fluxes is shown in the right hand panels of the same two plates. Here it can be seen that a very high quality image is produced at $1.7 \mathrm{keV}$, but that the $100 \mathrm{keV}$ image, in the hot tail of the proton energy distribution, is of marginal quality for the same aperture and exposure time, even though a much wider energy passband has been used.

\section{Recovery of Hot Plasma Ion Flux}

When we attempt the same ion flux recovery of the equatorial $1.7 \mathrm{keV}$ and $100 \mathrm{keV}$ fast atom images using the integral hydrogen density image, we find that this method is somewhat inaccurate within a substantial region of the inner magnetosphere for these equatorial views. The results of the operation for the $1.7 \mathrm{keV}$ case are shown in the right panels of Plate 3 for direct comparison with the true model flux image. The differences between true and recovered ion flux result from the highly structured nature of the modeled plasma region, wherein local time structures appear as asymmetric depth variations along the lines of sight that make up the image pixels.

In the case of the equatorial $100 \mathrm{keV}$ fast atom image, shown in the right hand panel of Plate 4 , the simple radial image adjustment is reasonably accurate except in the innermost region, where the difference in line-of-sight distributions of ions and cold atoms becomes apparent. The improved accuracy at $100 \mathrm{keV}$ stems from the greater uniformity of the energetic proton flux with local time and radius. In either case, it is clear that the equatorial fast atom images, when adjusted for the radial dependence of the hydrogen density, allow for qualitative discrimination between regions with "pancaked" and field-aligned PADs. In the following sections, we investigate the degree to which this can be used to quantitatively estimate the PADs in a specified region of space, using this simple image inversion method. 


\section{Integral lon Flux [L $[T E]^{-1}$}

\section{Recovered Ion Flux}
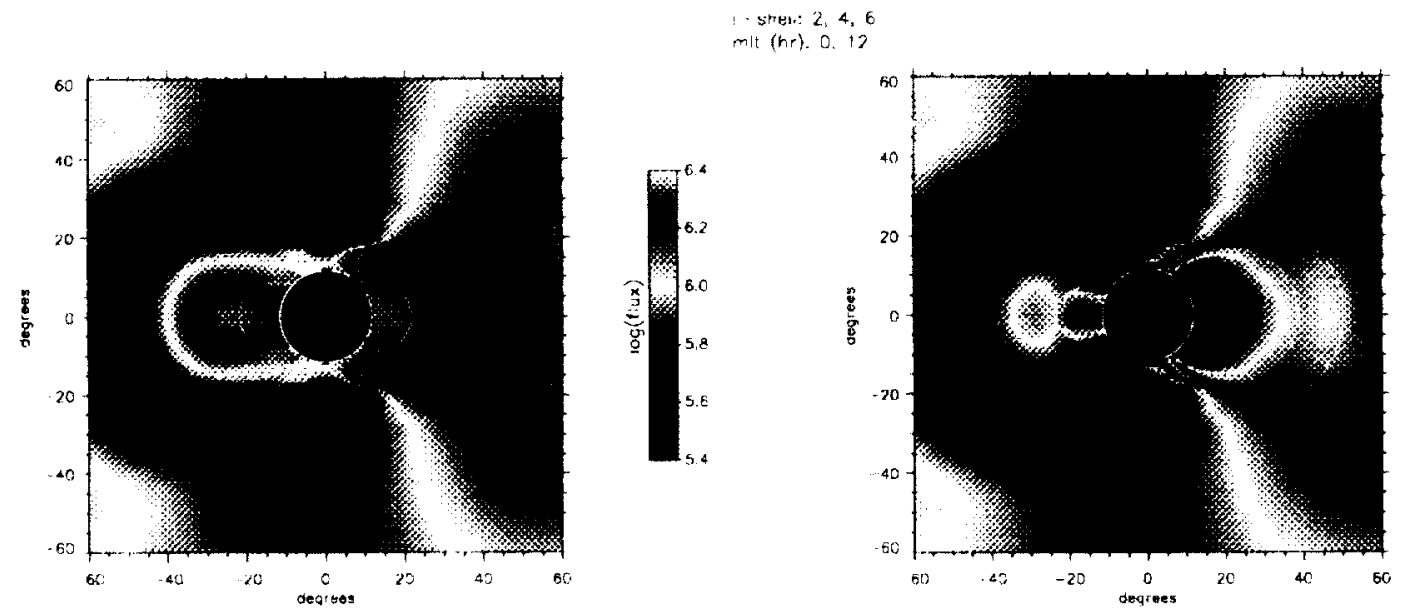

Plate 3. Similar to Plate 1, but for a vantage point located at the magnetic equatorial plane, at 1800 local time. Magnetic field lines at $L=2,4$, and 6 , at noon and midnight local times, have been superposed as a spatial reference system.

\section{Integral lon Flux $(L \Omega T E)^{-1}$}

\section{Recovered Ion Flux}
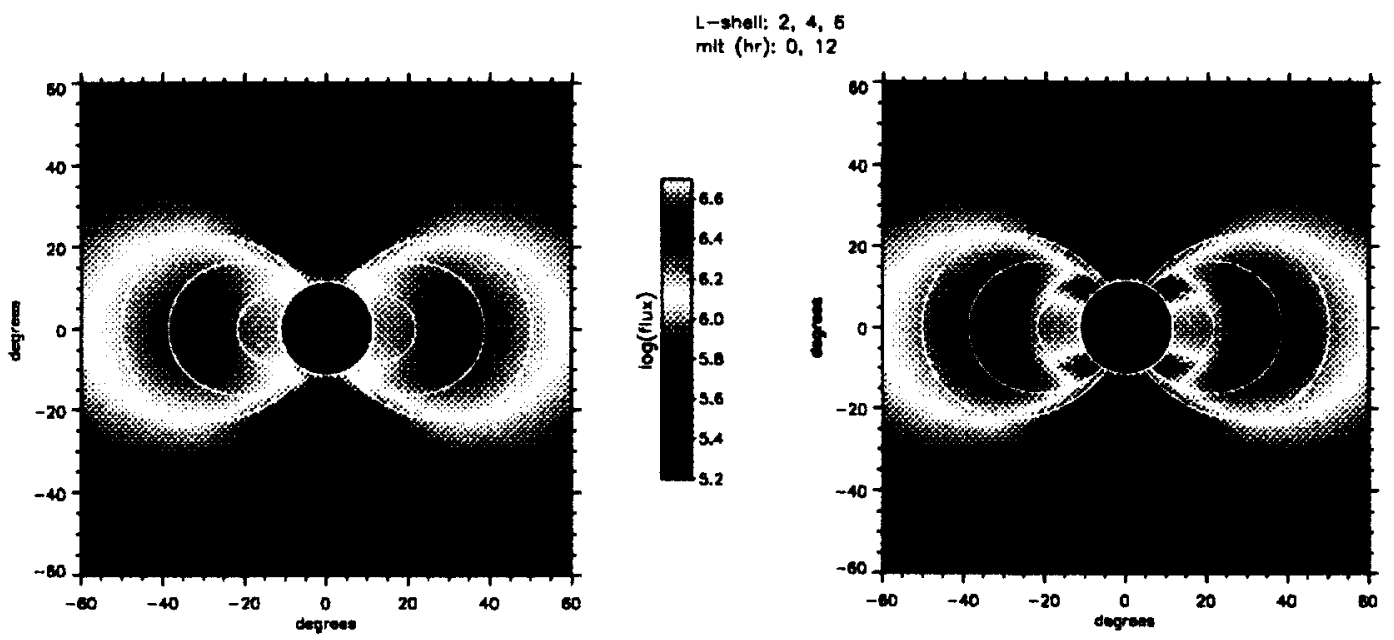

Plate 4. Similar to Plate 1, but for $100 \mathrm{keV}$ protons at 36 hours into the storm recovery and a vantage point like that of Plate 3, located at the magnetic equatorial plane, at 1800 local time. Magnetic field lines at $L=2,4$, and 6, at noon and midnight local times, have been superposed as a spatial reference system. 


\section{Neutral Atom Flux $\left(\mathrm{cm}^{2} \mathrm{sr} \mathrm{s} \mathrm{keV}\right)^{-1}$}

\section{Image Counts}

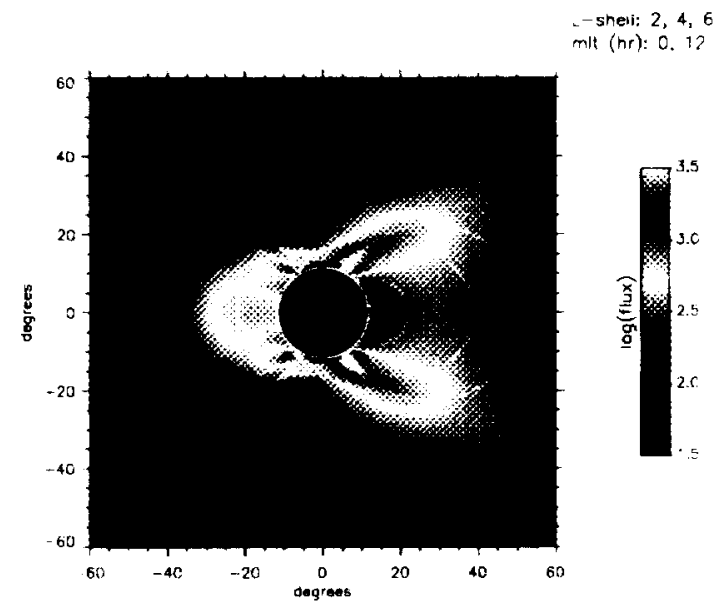

Plate 5. Similar to Plate 2, but for a vantage point located at the magnetic equatorial plane, 1800 local time. Magnetic field lines at $L=2,4$, and 6 at noon and midnight local times, have been superposed as a spatial reference system.

\section{Neutral Atom Flux $\left(\mathrm{cm}^{2} \mathrm{sr} \mathrm{s} \mathrm{keV}^{-1}\right.$}

\section{Image Counts}
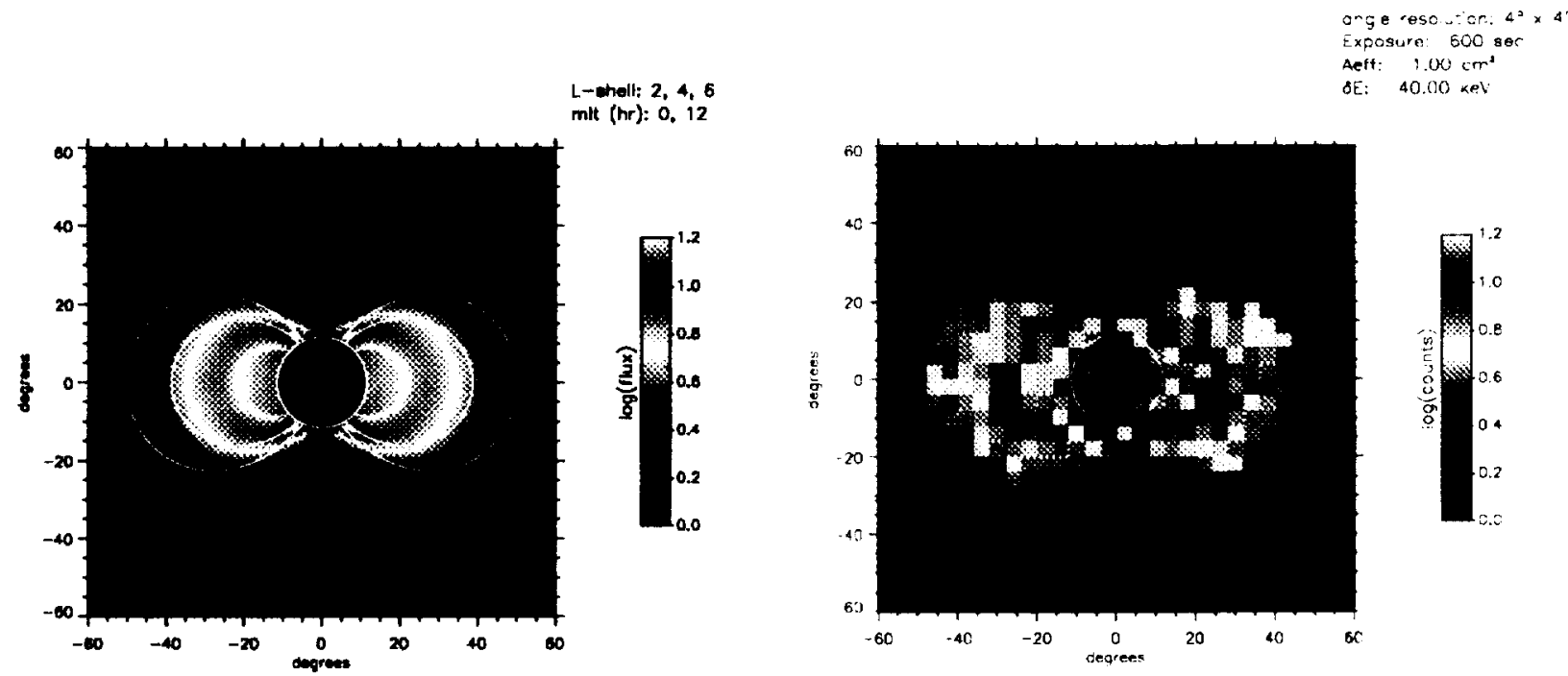

Plate 6. Similar to Plate 2, but for $100 \mathrm{keV}$ protons 36 hours into the storm recovery and a vantage point like that of Plate 3, located at the magnetic equatorial plane, 1800 local time. Magnetic field lines at $L=2,4$, and 6 at noon and midnight local times have been superposed as a spatial reference system. 


\section{Tangent-Shell Field Line}

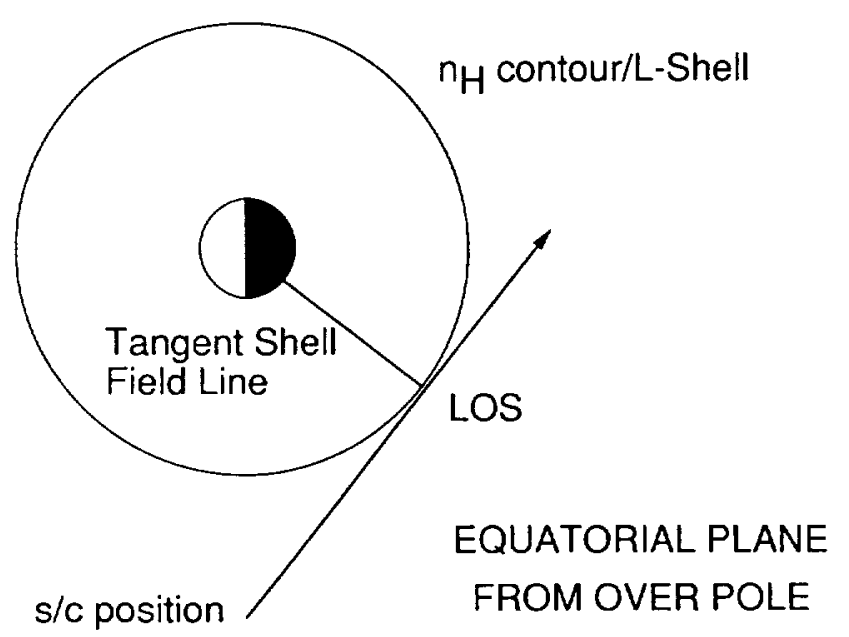

Fig. 1. Schematic illustration of the tangent-shell field line meridian, a concept needed for the interpretation of wide angle global imagery of fast atoms.

\section{QUANTITATIVE PADS FROM IMAGES}

\section{Tangent-Shell Field Line Meridian}

In discussing the images above, we have sometimes referred to them as if they represented the ion flux in the plane normal to the central line-of-sight. Since they are actually line-of-sight convolutions, this is not strictly true. They are more accurately described as best representing the ion flux within a "tangent-shell field line" meridian, which we define by the field line that is contained in a magnetic $L$-shell tangent to the current line-of-sight, and located at the point of tangency. This concept is closely related to the concept of an atmospheric limb, as illustrated in Figure 1. For example, an imager with a vantage point at $5 R_{E}$ at local dusk, viewing at an angle of 45 degree. from the line to the Earth, toward the nightshade, has a tangentshell field line lying at a local time of approximately 2100 hours, with an $L$ value of approximately 5.7.

Neutral atom images tend to be dominated by plasma conditions near the tangent-shell field line meridian, because this is the region along the line-of-sight where the neutral hydrogen density is strongly peaked. For a uniform distribution of ion flux, it is clear that the vast majority of emission along a particular line-of-sight originates near the tangent-shell field line. This is the basis of the method described in the following section, for associating an inferred PAD with a particular spatial region. The method will be compromised by strong departures from a uniform distribution of storm-time plasma, in which plasma regions far from the tangent-shell field line have dominant contri- butions to the line-of-sight convolution for a given pixel region.

\section{Spatially Uniform Storm Plasma}

As a simple demonstration of this technique, we use a spatially uniform storm plasma to compute normalized brightness dependencies upon geomagnetic latitude and $L$ value of field lines, and upon the PAD exponent, $n$, where the PAD is assumed to be well-described by the function $\sin ^{n}\left(\alpha_{e}\right)$, with $\alpha_{e}$ the equatorial pitch angle, and $n$ a real exponent. The procedure is to compute the fast neutral atom image based on the uniform storm plasma with specified $n$ 's. Tangent-shell field lines are then overlaid on the images, and a set of brightness versus latitude curves is developed for each value of $n$. The set of curves then constitutes a renormalizable calibration between brightness curves and PADs. Plate 7 (left panel) shows the computed flux image for a uniform storm plasma spatial distribution with PAD index, $n=1$. The right panel of Plate 7 shows the set of flux curves computed for the indicated $L=4$ field line and the indicated set of PAD indexes, $n$. The curves can be readily seen to incorporate the cold geocoronal hydrogen distribution, having footpoint brightness that in general are larger than equatorial brightnesses, except for strongly "pancaked" PADs, i.e., those with large positive $n$.

\section{PAD RESULTS}

The superposition of specific fast atom image brightness curve upon the family of curves shown in Plate 7 allows inference of the PAD in the tangent-shell region that dominates the image. More generally, we would envision the parameterization of the curves and a fitting process whereby the observed image brightness would lead to an inferred set of PAD parameters. As a demonstration of this technique, Figure 2 shows two case studies from the images previously shown in Plates 5 and 6.

In the left panel of Figure 2, the $1.7 \mathrm{keV}$ image data from dayside and nightside, along the $L=4$ field line, are superposed on the family of curves from Plate 7 . It can be seen that there are notable differences between the shape of the data and the curves, but that qualitatively, the difference in pitch angle distribution is clearly evident. The equatorial (center) parts of the data traces reflect the true PADs significantly better than the higher latitude parts of the data traces, because of increasing contributions from regions well away from the tangent-shell meridian, in the inner parts of the images.

In the right panel of Figure 2, the $100 \mathrm{keV}$ image data from the nightside are superposed on the same set of curves. Here there is remarkably good agreement between the shape of the modeled curves and that of the simulated data trace from the fast atom image. Moreover, the interpolated value of the PAD index $n$ that is inferred from the position of the simulated data on the set of curves, is in 

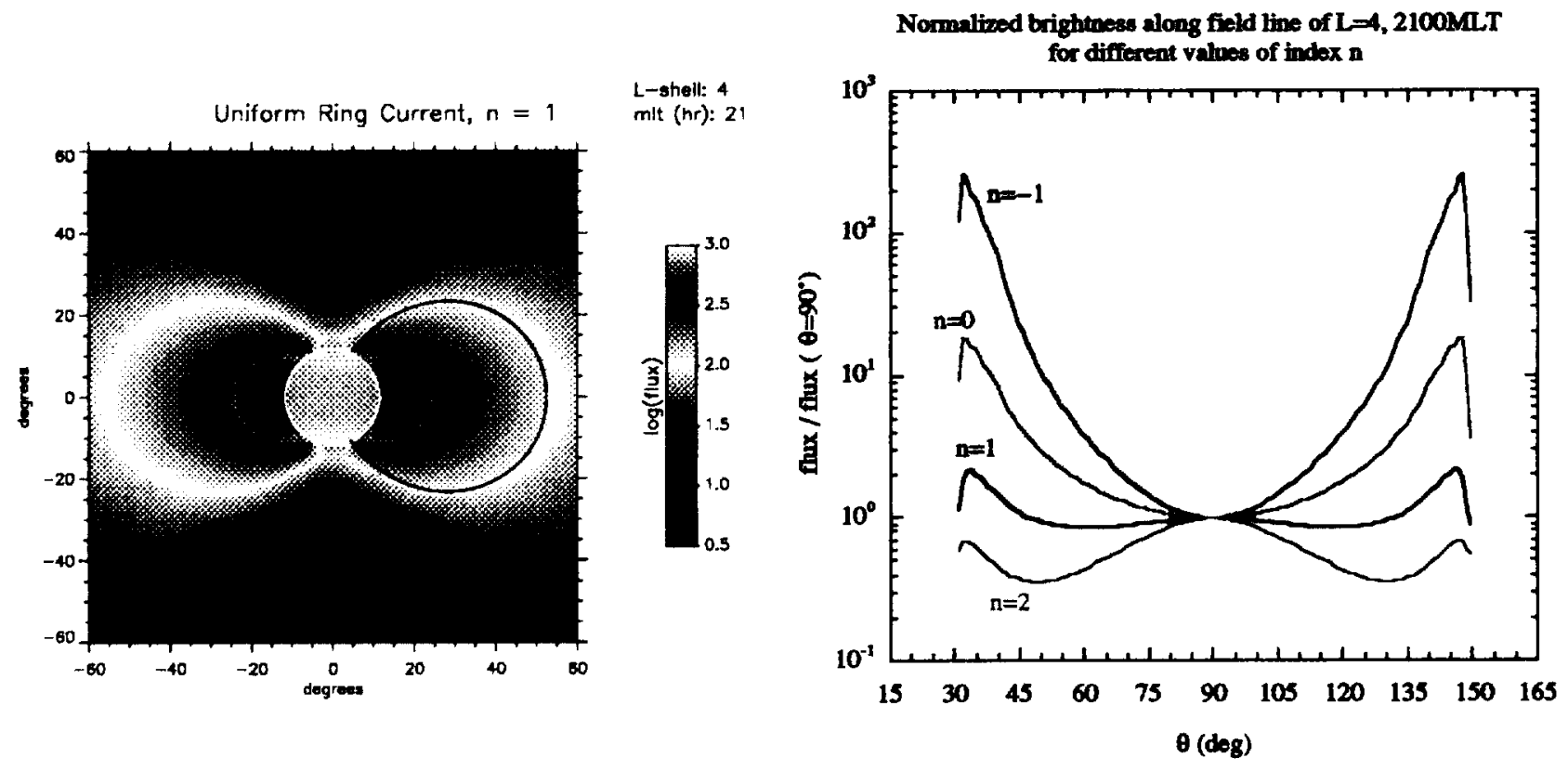

Curve of $n=1$ is extracted from the image on the left

Plate 7. Left panel: the fast atom flux image generated by a uniform distribution of flux with a pitch angle distribution given by $\sin ^{n}\left(\alpha_{e}\right)$, where $n=1$ throughout the modeled region. Right panel: the flux image brightness as a function of magnetic latitude along the $L=4$ field line indicated in the left panel, for the indicated values of $n$ in the (uniform) PAD.
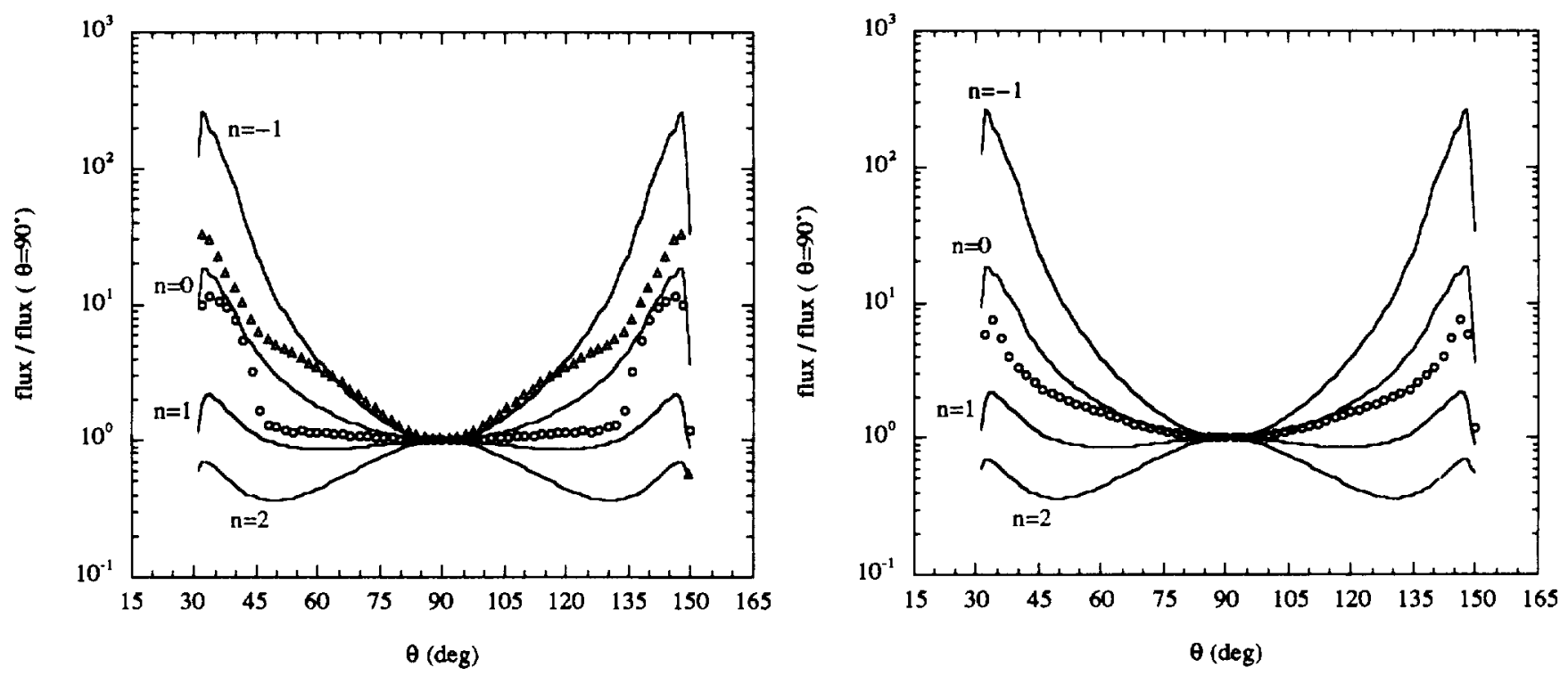

- $\quad 1.7 \mathrm{keV} \mathrm{H}^{+}$at $1500 \mathrm{MLT}$, True $\mathrm{n}=1.2$

- $1.7 \mathrm{keV} \mathrm{H}^{+}$at $2100 \mathrm{MLT}$, True $\mathrm{n}=-0.5$

- $100 \mathrm{keV} \mathrm{H}^{+}$at $2100 \mathrm{MLT}$, True $n=0.47$

Fig. 2. Comparison of simulated image data with the tangent-shell field line flux curves of Plate 7. Left panel: case of $1.7 \mathrm{keV}, 6$ hours recovery phase. Right panel: case of $100 \mathrm{keV}, 36$ hours recovery phase. 
good agreement (to one significant digit) with the true PAD in the tangent shell meridian region. The $100 \mathrm{keV}$ case is clearly much more successful than the $1.7 \mathrm{keV}$ case, because the nature of the $100 \mathrm{keV}$ plasma distribution is much closer to that of the hot plasma used to generate the image flux curves; that is, more uniform in local time and radius.

\section{SUMMARY/CONCLUSIONS}

We have used a newly developed three-dimensional model of the recovery phase storm plasma to explore the signatures of pitch angle distributions in fast atom imagery of hot plasmas. We find that fast atom images are significantly influenced by the spatio-temporal characteristics of pitch angle distributions included in this model. Said differently, we have shown that global fast atom images contain detailed information about the pitch angle distribution of ions within the imaged regions. In the process, it has become clear that fast atom images cannot be accurately deconvolved without attention to PAD effects, since they can otherwise masquerade as spatial distribution effects.

When PAD effects are properly treated, magnetospheric hot plasma imaging promises a significant new capability to sense the global effects of microphysical processes. This would be done by monitoring the global distribution of plasma wave intensities through their diffusive effects on PADs. We have shown that the assumption of spatial uniformity provides a useful tool for quickly estimating PADs and identifying morphological features of the PAD in space. PAD inferences using this tool are best for high energy ions that are relatively uniform in local time, on $L$ shells where radial gradients are weak. However, useful qualitative inferences can be made almost trivially using this method, and gross parameters of the ion flux distribution can be estimated as inputs to a more rigorous iterative inversion procedure.

The limited successes of the simple techniques illustrated here suggest an optimistic view of the prospects for quantitative image inversion. Sophisticated deconvolution methods, such a maximum entropy analysis and Bayesian statistics, are now being brought to bear on the problem, and very encouraging results are being obtained. This optimism is bolstered by the empirical fact that readily interpreted features of the images are subject to straightforward modeling inferences.

Acknowledgments. The authors have benefited from stimulating discussions with D. L. Gallagher, who has contributed to the coding of the line-of-sight integration, and applied it to the simulation of images of cold $\mathrm{He}^{+}$plasma in scattered solar $30.4 \mathrm{~nm}$ EUV light. We are also indebted to $M$. A. Sloan of Computer Sciences Corp., who has contributed programming discipline and documentation to the analysis and display of these results. M.-C. Fok was a Resident Research Associate of the National Research Council at Marshall Space Flight Center during this work, which was also was supported by Marshall Space Flight Center and by the NASA Space Physics Division under UPN 433-90-00. Work at Auburn University was supported by grant NAG8-147 from the NASA JOVE program, and by grant NGT-51046 from the NASA/Marshall Space Flight Center Graduate Student Researcher Program.

\section{REFERENCES}

Fok, M.-C., T. E. Moore, J. U. Kozyra, G. C. Ho, and D. C. Hamilton, A three-dimensional ring current decay model, $J$. Ceophys. Res. 100, 9619, 1995.

Hamilton, D. C., G. Gloeckler, F. M. Ipavich, W. Studemann, B. Wilken, and G. Kremser, Ring current development during the great geomagnetic storm of February, 1986, J. Geophys. Res. $93,14,343,1988$

Moore, K. R., D. J. McComas, H. O. Funsten, and M. F. Thomsen, Low energy neutral atoms in the Earth's magnetosphere modeling, in Instrumentation for Magnetospheric Imagery, ed. by S. Charkrabarti, p. 51, Proc. SPIE, p. 1744. (also submitted to Optical Science), 1992.

Rasmussen, C. E., S. M. Guiter, and S. G. Thomas, Two-dimensional model of the plasmasphere: refilling time constants, Planet. Space Sci. 41, p. 35, 1993.

Roelof, E. C., D. G. Mitchell, and D. J. Williams, Energetic neutral atoms $(\sim 50 \mathrm{keV})$ from the ring current: IMP $7 / 8$ and ISEE 1, J. Geophys. Res. 90, p. 10,991, 1985.

Williams, D. J., E. C. Roelof, and D. G. Mitchell, Global Magnetospheric Imaging, Rev. Geophvs. 30(3), 183, 1992.

T. E. Moore and M.-C. Fok, Space Sciences Laboratory, NASA Marshall Space Flight Center, Huntsville, AL 35812.

J. D. Perez and J. P. Keady, Auburn University, Auburn, AL 36849. 